PROCEEDINGS OF THE

AMERICAN MATHEMATICAL SOCIETY

Volume 128, Number 4, Pages 1177-1183

S 0002-9939(99)05253-3

Article electronically published on October 18, 1999

\title{
GEOMETRY OF A CROSSED PRODUCT
}

\author{
IGOR NIKOLAEV
}

(Communicated by David R. Larson)

\begin{abstract}
We introduce a continuous dimension function $\alpha: \bullet \rightarrow \mathbb{R}$ on the Grothendieck group $K_{0}$ over the crossed product $C^{*}$-algebra $C(X) \rtimes_{\phi} \mathbb{Z}$. The function $\alpha$ has an elegant geometry: on every minimal flow $\phi^{t}$ it takes the value of the "rotation number" of $\phi^{t}$; such a problem was posed in 1936 by
\end{abstract} A. Weil.

\section{Algebraic introduction}

Let 1 be a Cantor set and let $C(X)$ be a $C^{*}$-algebra of continuous, complexvalued functions on $X$. Let $\phi: X \rightarrow X$ be a minimal homeomorphism of $X$. A crossed product $C^{*}$-algebra $C(X) \rtimes_{\phi} \mathbb{Z}$ is a universal $C^{*}$-algebra generated by $C(X)$ and a unitary operator $u$ such that $u f u^{*}=\phi(f)$ for all $f \in C(X)$.

A bulk of important information on $C(X) \rtimes_{\phi} \mathbb{Z}$ can be read-off from its Grothendieck's group $K_{0}$ which is often calculable and handier in work. Due to Elliott [4] we know that for the approximate finite $(\mathrm{AF}) C^{*}$-algebras its (ordered) $K_{0}$-group encodes all the information on AF-algebra. Unfortunately $C(X) \rtimes_{\phi} \mathbb{Z}$ is not AF, but can be embedded into an AF-algebra whose $K_{0}$-group is order isomorphic to the $K_{0}$-group of our crossed product algebra; this deep result belongs to Putnam [11.

Can one 'compute' $K_{0}$ for the concrete crossed product $C^{*}$-algebras? In the important case of the irrational rotation algebra $A_{\alpha}$ (i.e. when $X=S^{1}$ and $\phi(x)=$ $x+\alpha)$ the answer is yes. As it shows one beautiful result of Effros and Shen, the group $K_{0}$ is nicely connected with the 'geometry' of $\alpha$.

Theorem 1 ([3]). Let $A_{\alpha}$ be an irrational rotation $C^{*}$-algebra. Suppose that the real number $\alpha$ has a continued fraction expansion

$$
\alpha=a_{0}+\frac{1}{a_{1}+\frac{1}{a_{2}+\ldots}} \stackrel{\text { def }}{=}\left[a_{0}, a_{1}, a_{2}, \ldots\right] .
$$

Received by the editors November 14, 1997 and, in revised form, June 17, 1998.

1991 Mathematics Subject Classification. Primary 46L40, 57R30, 58F10.

Key words and phrases. Dimension group, continued fraction, minimal flow.

${ }^{1}$ No motivation to such a setting is furnished for the moment; to comfort the reader at this stage we mention that it comes from the study of minimal flows on the closed orientable surfaces of genus $g>1$ and the interval exchange transformations over the unit circle. As a preliminary reading we recommend a chapter on the irrational rotation algebra from the book of Davidson [2] and material on the Giordano-Putnam-Skau's theory scattered in 5, 10] and 11. See also the last section of the present notes. 
Let $\varphi_{n}$ be a composition of isometries of the lattice $\mathbb{Z}^{2} \subset \mathbb{R}^{2}$ :

$$
\varphi_{n}=\left(\begin{array}{cc}
a_{0} & 1 \\
1 & 0
\end{array}\right) \cdots\left(\begin{array}{cc}
a_{n} & 1 \\
1 & 0
\end{array}\right) .
$$

Then $A_{\alpha}$ can be embedded into an AF-algebra whose $K_{0}$-group is order isomorphic to the direct limit of ordered abelian groups $P_{\alpha}=\lim _{n \rightarrow \infty}\left(\mathbb{Z}^{2}, \varphi_{n}\right)$. Moreover, if $\alpha=\left[a_{0}, a_{1}, \ldots\right]$ and $\beta=\left[b_{0}, b_{1}, \ldots\right]$ are two irrational numbers, then $P_{\alpha}$ and $P_{\beta}$ are isomorphic if and only if $a_{m+k}=b_{m}$ for an integer number $k \in \mathbb{Z}$. (In other words, the irrational numbers $\alpha, \beta$ are modular equivalent: $\beta=(a \alpha+b) /(c \alpha+d)$, $a d-b c= \pm 1$, where $a, b, c, d \in \mathbb{Z}$ are integer numbers.)

An inspiration behind this note was to extend Theorem 1 to the case when $X$ is a Cantor set and $\phi$ is a minimal homeomorphism on $X$. Clearly, as a trophy one would expect a rotation number, $\alpha$, which generalizes the Poincaré rotation number to the case of flows on the closed surfaces of genus $g>1$; this is exactly the Weil's problem of 1936; cf. 12]. We prove more, by showing that (modular equivalent) $\alpha$ 's are complete invariants of the topological conjugacy of minimal flows and (up to Morita equivalence) of the corresponding crossed product $C^{*}$-algebras.

\section{Calculation of $K_{0}\left(C(X) \rtimes_{\phi} Z\right)$}

Let $\phi: X \rightarrow X$ be a minimal homeomorphism on the Cantor set $X$. It was shown in [11] that the suspension of $\phi$ is a minimal foliation 2 on the closed manifold $M$ of dimension $(\operatorname{dim} X)+1$. For the sake of simplicity we let further $\operatorname{dim} X=1$ so that $M$ is a closed 2-dimensional manifold with a minimal flow $\phi^{t}$ on it. The Cantor set $X \subset S^{1}$ in this case corresponds to the intersection of the closure of a (discontinuous) geodesic line $\gamma$ in the metric of constant negative curvature on $M$ with the closed transversal $S^{1}$; cf. [10.

With no exception, $M$ is given by its universal covering representation $(\mathbb{H}, \Gamma)$ where $\mathbb{H}=\{z=x+i y \in \mathbb{C}: y \geq 0\}$ is the Lobachevsky (half-) plane endowed with the hyperbolic metric $d s=|d z| / y$ and $\Gamma$ is a discontinuous group of isometries of $\mathbb{H}$. We identify $\Gamma$ with the principal congruence group $\Gamma(n)= \pm\left(\begin{array}{ll}1 & 0 \\ 0 & 1\end{array}\right) \operatorname{Mod} n, n \in \mathbb{N}$.

It is well known that on $\mathbb{H}$ the geodesic lines are represented either by semicircles or straight lines orthogonal to the absolute $y=0$. By the ergodicity of geodesic flow 3 over $M$ every geodesic line in $\mathbb{H}$ can be 'iterated' arbitrarily close by the images $\Gamma_{i}(p)$ of a geodesic line $p$ belonging to a certain set of full Lebesgue measure at the absolute. (This is exactly an Approximationssatz established by Myrberg [9] in 1931.) Now we are ready to introduce the following important quantity connected with the $C^{*}$-algebra $C(X) \rtimes_{\phi} \mathbb{Z}$ :

Definition 1. Let $\tilde{\gamma}$ be a covering of the geodesic line $\gamma$ at the Lobachevsky plane $\mathbb{H}$. Let $S_{0}$ be the fundamental polygon of the group $\Gamma$ bounded by the geodesic sides $a_{1}, b_{1}, \ldots, a_{2 g}, b_{2 g}$ which represent generators of the group $\pi_{1}(M)$. Let $\varepsilon_{1}, \varepsilon_{2}, \ldots$ be a monotone sequence of positive real numbers tending to zero. Let, finally, $T_{0}, T_{1}, T_{2}, \ldots$ be an infinite sequence of isometries $\Gamma(n)$ 's which approximate the geodesic line $\tilde{\gamma}$ by the images of a fixed geodesic side of $S_{0}$ with the accuracy

\footnotetext{
${ }^{2}$ Foliation (with singularities) is called minimal if every non-singular leaf of it is dense on the manifold $M$.

${ }^{3}$ Regarding this important concept we refer the reader to the monograph of Hopf $[\underline{6}]$.
} 
$\varepsilon_{0}, \varepsilon_{1}, \varepsilon_{2}, \ldots$ By a rotation number of the $C^{*}$-algebra $C(X) \rtimes_{\phi} \mathbb{Z}$ one understands a real number $\alpha=\left[a_{0}, \ldots, a_{n_{0}}, a_{n_{0}+1}, \ldots, a_{n_{1}}, \ldots\right]$, where

$$
T_{0}=\left(\begin{array}{cc}
a_{0} & 1 \\
1 & 0
\end{array}\right) \ldots\left(\begin{array}{cc}
a_{n_{0}} & 1 \\
1 & 0
\end{array}\right) ; \quad T_{1}=\left(\begin{array}{cc}
a_{n_{0}+1} & 1 \\
1 & 0
\end{array}\right) \ldots\left(\begin{array}{ll}
a_{n_{1}} & 1
\end{array}\right) ; \ldots
$$

is the Minkowski decomposition (cf. [8]) of the positive integer matrices $T_{i}$ 's.

(The 'geometric meaning' of $\alpha$ is an inclination of the geodesic line $\tilde{\gamma}$ relative to a lattice $\mathbb{L} \subset \mathbb{H}$ formed by the vertices of the fundamental polygons $\Gamma\left(S_{0}\right)$ in $\mathbb{H}$. Clearly, choosing another side from the collection $a_{1}, b_{1}, \ldots, a_{2 g}, b_{2 g}$, one obtains $\alpha$ 's which differ from each other by a constant.)

Connected with the rotation number $\alpha$ there is a dimension group $\left(L, L^{+}\right)$nested at the abelian lattice $\mathbb{L} \simeq \mathbb{Z}^{2 g}$. To introduce the latter, let us consider an infinite sequence of the positive cones $L_{0}^{+}, L_{1}^{+}, \ldots$ which correspond to the sequence of hyperplanes $L_{0}, L_{1}, \ldots$ in $\mathbb{R}^{2 g}$. (These hyperplanes are the 'lifting' to $\mathbb{R}^{2 g}$ of the approximants $T_{0}, T_{1}, \ldots$ of the geodesic $\tilde{\gamma}$ in $\mathbb{H}$.) We define the dimension group $\left(L, L^{+}\right)$as a limit of the ordered abelian groups $\left(L_{0}, L_{0}^{+}\right),\left(L_{1}, L_{1}^{+}\right), \ldots$

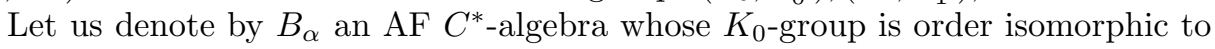
$\left(L, L^{+}\right)$. The following embedding lemma is used in calculation of the Grothendieck's group $K_{0}$.

Lemma 1. There exists an embedding $i: C(X) \rtimes_{\phi} \mathbb{Z} \rightarrow B_{\alpha}$ such that

$$
i_{*}: K_{0}\left(C(X) \rtimes_{\phi} \mathbb{Z}\right) \rightarrow K_{0}\left(B_{\alpha}\right)
$$

is an isomorphism of the ordered abelian groups.

Proof. The proof is based on the 'tower' construction; cf. Davidson [2]. Let $Y \subset X$ be a closed subset of the Cantor set $X$. Let $\phi: X \rightarrow X$ be a minimal homeomorphism on $X$. Define an integer-valued function $\lambda: Y \rightarrow \mathbb{Z}$ by the formula

$$
\lambda(y)=\min \left\{n \geq 1: \phi^{n}(y) \in Y, y \in Y\right\} .
$$

Note that $\lambda$ is a well-defined function on $Y$ and takes only finite number of values on it. We let $\lambda(Y)=\left\{J_{1}, J_{2}, \ldots, J_{K}\right\}$ be an ordered sequence of positive integers $J_{1}<J_{2}<\cdots<J_{K}$. Finally, for $k=1,2, \ldots, K$ and $j=1,2, \ldots, J_{K}$ we define the closed-open ("clopen") subsets $Y(k, j)=\phi^{j}\left(\lambda^{-1}\left(J_{k}\right)\right)$. The collection $\{Y(k, j) \mid j=$ $\left.1, \ldots, J_{k}\right\}$ is called a tower of height $J_{k}$. Connected with every 'tower' there is a multi-matrix $C^{*}$-algebra

$$
M_{J_{1}} \oplus M_{J_{2}} \oplus \cdots \oplus M_{J_{K}}
$$

whose matrix units $e_{i j}^{(k)}$ are chosen accordingly with the formula $e_{i j}^{(k)}=\chi_{Y(k, i)} u^{i-j}$. (Here $u$ is unitary operator on the $C^{*}$-algebra $C(X)$ and $\chi_{Y(k, i)}$ is the characteristic function of the set interval $Y(k, i)$.)

Now fix $K=2 g$, where $g$ is genus of the underlying surface $M$. Taking a chain of inclusions $Y_{0} \supset Y_{1} \supset \ldots$ such that $\bigcap Y_{i}=\{y\}$ is a point in $X$, one gets a sequence of towers converging to an AF $C^{*}$-algebra $A_{\{y\}}$. Let $P_{\alpha}$ be a dimension group corresponding to the AF-algebra $A_{\{y\}}$. By $\varphi_{i}$ 's we denote positive integer unitary 
operators acting on the lattice $\mathbb{Z}^{2 g}$. Consider the following commutative diagram:

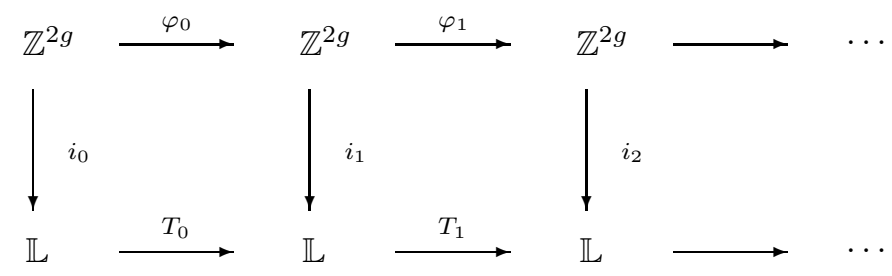

where $T_{0}, T_{1}, T_{2}, \ldots$ is the defining sequence for the rotation number $\alpha$. Since $\mathbb{Z}^{2 g} \simeq$ $\mathbb{L}$, there exists an order isomorphism $i_{n}: \mathbb{Z}^{2 g} \rightarrow \mathbb{L}$ such that $\varphi_{m} \circ i_{m+1}=i_{m} \circ T_{m}$. It remains to take the limit $i=\lim _{m \rightarrow \infty} i_{m}$ which closes the infinite commutative diagram. Therefore, $P_{\alpha}$ is order isomorphic to the dimension group $\left(L, L^{+}\right)$of the lattice $\mathbb{L}$.

The rest of the proof follows from Putnam's theorem (Theorem 4.1 of [11]) which says that $C(X) \rtimes_{\phi} \mathbb{Z}$ is embeddable into the AF-algebra $A_{\{y\}}$ so that $K_{0}\left(C(X) \rtimes_{\phi} \mathbb{Z}\right) \simeq P_{\alpha}$. Lemma 1 is proved.

Corollary 1. Let $C(X) \rtimes_{\phi} \mathbb{Z}$ be a $C^{*}$-algebra generated by the minimal homeomorphism $\phi$ on the Cantor set $X$. Suppose that the rotation number of $C(X) \rtimes_{\phi} \mathbb{Z}$ has a continued fraction expansion $\alpha=\left[a_{0}, a_{1}, a_{2}, \ldots\right]$. Let, finally, $\varphi_{n}$ be a composition of isometries of the lattice $\mathbb{L} \subset \mathbb{H}$ :

$$
\varphi_{n}=\left(\begin{array}{cc}
a_{0} & 1 \\
1 & 0
\end{array}\right) \ldots\left(\begin{array}{cc}
a_{n} & 1 \\
1 & 0
\end{array}\right) .
$$

Then the $K_{0}$-group of $C(X) \rtimes_{\phi} \mathbb{Z}$ is order isomorphic to a dimension group $P_{\alpha}=$ $\lim _{n \rightarrow \infty}\left(\mathbb{L}, \varphi_{n}\right)$. Moreover, if $C(X) \rtimes_{\phi} \mathbb{Z}$ and $C(X) \rtimes_{\psi} \mathbb{Z}$ are two crossed product $C^{*}$ algebras with the rotation numbers $\alpha=\left[a_{0}, a_{1}, \ldots\right]$ and $\beta=\left[b_{0}, b_{1}, \ldots\right]$ respectively, then they are Morita equivalent if and only if $a_{m+k}=b_{m}$ for an integer number $k \in \mathbb{Z}$. The latter means that the irrational numbers $\alpha$ and $\beta$ are modular equivalent.

Proof. The first part follows from the Lemma 1 and Definition 1. The necessary conditions in the second part are an implication of the basic property of continued fractions. The sufficient conditions are less evident; they follow from GiordanoPutnam-Skau's theory; see e.g. Theorem 2.1 of [5].

\section{Rotation NUMBERS OF Minimal FLOWS}

Let $M$ be a closed orientable surface of genus $g>1$. With a little abuse of notation a $C^{\infty}$ flow $\phi^{t}$ on $M$ will be called minimal if every non-stationary trajectory of $\phi^{t}$ is everywhere dense in $M$ and the set Sing $\phi^{t}$ consists of $2 m$-separatrix saddles, $m \geq 2$. Let $S^{1}$ be a cross-section to the minimal flow $\phi^{t}$ on $M$. Let $\phi: S^{1} \rightarrow S^{1}$ be the first return mapping induced on $S^{1}$ by $\phi^{t}$. We have a partition of $S^{1} \cong[0,1)$ into the semi-intervals $I_{i}=\left[x_{i-1}, x_{i}\right)$ where $0=x_{0}<x_{1}<\cdots<x_{r}=1$ are the points where the separatrices of $\phi^{t}$ intersect first with $S^{1}$. $\phi$ is known to permute the order of $I_{i}$ on $S^{1}:\left(I_{1}, I_{2}, \ldots, I_{r}\right) \mapsto\left(I_{\pi(1)}, I_{\pi(2)}, \ldots, I_{\pi(r)}\right)$, where $\pi$ is the corresponding permutation group. 
By the minimality of flow the set of points $\Lambda=\left\{\phi^{n}\left(x_{i}\right) \mid 0 \leq i<r ; n \in \mathbb{Z}\right\}$ is everywhere dense in $S^{1}$. If we "blow-up" each separatrix of the initial flow $\phi^{t}$, then each point in $\Lambda$ will split into two points, $y_{1}<y_{2}$, at $S^{1}$. Throwing-out all the open intervals $\left(y_{1}, y_{2}\right)$ 's one comes to the Cantor set $X$ on $S^{1}$. $X$ corresponds to the intersection of $S^{1}$ with the closure of the discontinuous geodesic line $\tilde{\gamma}$ built upon the flow $\phi^{t}$; cf. Aranson et al. [1]. One defines a homeomorphism $\phi: X \rightarrow X$ so that it coincides on the set $S^{1} \backslash \Lambda$ with the initial mapping $\phi: S^{1} \rightarrow S^{1}$.

A $C^{*}$-algebra of continuous complex-valued functions on $X$ crossed by the action of powers of $\phi$ is $*$-isomorphic to $C(X) \rtimes_{\phi} \mathbb{Z}$; cf. Putnam [1]. A real number $\alpha$ connected with the $C^{*}$-algebra $C(X) \rtimes_{\phi} \mathbb{Z}$ is called a rotation number of the minimal flow $\phi^{t}$. It was shown in [10] that, up to a relation which identifies modular equivalent numbers, $\alpha$ is uniquely defined by the flow $\phi^{t}$. Moreover, $\alpha$ is a complete 'modular' invariant of the topological equivalence 4 of the minimal flows on $M$.

Theorem 2. Let $\phi^{t}$ and $\psi^{t}$ be two minimal flows on the surface $M$ whose rotation numbers are $\alpha$ and $\beta$, respectively. Then the flows $\phi^{t}$ and $\psi^{t}$ are topologically equivalent if and only if $\alpha$ and $\beta$ are modular equivalent real numbers.

Proof. Necessary conditions. Suppose that two minimal flows $\phi^{t}, \psi^{t}$ are topologically equivalent: $\psi^{t}=h \phi^{t} h^{-1}$. Let $\gamma_{\phi}, \gamma_{\psi}$ be the respective geodesics lines, which define the equivalence classes of these flows; cf. Aranson et al. [1]. Let, finally, Clos $\gamma_{\phi}$, Clos $\gamma_{\psi}$ be the closures of the geodesic lines $\gamma_{\phi}, \gamma_{\psi}$ on the Lobachevsky plane $\mathbb{H}$.

Take arbitrary connected components, $\tilde{\gamma}_{\phi}$ and $\tilde{\gamma}_{\psi}$, in the preimages of $\gamma_{\phi}$ and $\gamma_{\psi}$ on $\mathbb{H}$. Since flows $\phi^{t}$ and $\psi^{t}$ are equivalent, both $\tilde{\gamma}_{\phi}$ and $\tilde{\gamma}_{\psi}$ belong to the same set Clos $\gamma_{\phi} \equiv$ Clos $\gamma_{\psi}$ at $\mathbb{H}$. This means that there exists an isometry $h \in \Gamma$ such that $\tilde{\gamma}_{\psi}=\tilde{h} \tilde{\gamma}_{\phi} \tilde{h}^{-1}$. By the basic property of continued fractions the rotation numbers $\alpha$ and $\beta$ are modular equivalent.

Sufficient conditions. Let us assume now that $\alpha$ and $\beta$ are modular equivalent. In constructing the conjugacy homeomorphism $h: \psi^{t}=h \circ \phi^{t} \circ h^{-1}$, the following lemma is instrumental.

Lemma 2. Suppose that the flows $\phi^{t}$ and $\psi^{t}$ are as specified before. Let $\alpha \simeq \beta$ be their modular equivalent Artin's numbers. Then, $\phi^{t}$ and $\psi^{t}$ have mutually homotopic, transversal cycles, $C_{\phi}$ and $C_{\psi}$, intersecting the respective geodesics lines, $\gamma_{\phi}$ and $\gamma_{\psi}$.

Proof of lemma. Let $\gamma_{\phi}$ and $\gamma_{\psi}$ be as before. Since $\alpha \simeq \beta$, there exists a connected component in the preimages of $\gamma_{\phi}$ and $\gamma_{\psi}$ on the universal covering, such that $\tilde{\gamma}_{\phi}=\tilde{\gamma}_{\psi}=\tilde{\gamma}$. Let us further denote by $\tilde{\phi}^{t}$ and $\tilde{\psi}^{t}$ the corresponding flows on the covering surface $\tilde{M}$.

Every transitive flow on $M$ admits a transversal cycle; see Aranson et al. [1]. Take one of our flows, say $\phi^{t}$; clearly, it is transitive on $M$. Denote by $C_{\phi}$ the corresponding transversal cycle. Let $\tilde{C}$ be a connected component of its lifting to the universal covering surface $\tilde{M}$. Let us show that $\tilde{C}$ is a transversal cycle to the flow $\tilde{\psi}^{t}$, as well.

Indeed, suppose to the contrary, that $p \in \tilde{C}$ is a contact point to the flow $\tilde{\psi}^{t}$. By the assumption, $\tilde{\gamma}_{\psi}$ is an everywhere dense trajectory of the flow $\tilde{\psi}^{t}$. In particular, it approximates deliberately close the trajectory $l(p)$ of the flow $\tilde{\psi}^{t}$, issued through

\footnotetext{
${ }^{4} \mathrm{By}$ a topological equivalence we understand the orbital topological equivalence between the flows, which identifies flows with the same 'orbit structure'; cf. Aranson et al. [1].
} 
the contact point $p$. But $\tilde{\gamma}_{\psi}=\tilde{\gamma}_{\phi}=\tilde{\gamma}$, so that the flow $\tilde{\phi}^{t}$ must have a contact point with $\tilde{C}$. A contradiction. The lemma is proved.

The idea now is to reduce the conjugacy problem on the whole $M$ to the conjugacy problem between the first return mappings on the transversal cycles $C_{\phi}$ and $C_{\psi}$. This will be done in the following steps. (Here again, we refer the reader for the illustrations and details to the monograph [1].)

Let $p_{1}, p_{2}, \ldots, p_{8 g-8} \in \tilde{C}_{\phi}$ and $q_{1}, q_{2}, \ldots, q_{8 g-8} \in \tilde{C}_{\psi}$, where $g$ is the genus of $M$, be the points in which the separatrices of the saddle points hit the respective transversal cycles. (We conjectured, for simplicity, that all the saddle points are 4 -separatrix.) On account of the orientation of the flows $\tilde{\phi}^{t}$ and $\tilde{\psi}^{t}$, we fix the mapping $\tilde{h}$ :

$$
\tilde{h}\left(p_{1}\right)=q_{1}, \quad \tilde{h}\left(p_{2}\right)=q_{2}, \ldots, \tilde{h}\left(p_{8 g-8}\right)=q_{8 g-8} .
$$

(It should be understood that the construction enrolls in a fixed fundamental polygon, $S_{0}$, of the universal covering surface $\tilde{M}$.) Both of the geodesic lines $\tilde{\gamma}_{\phi}$ and $\tilde{\gamma}_{\psi}$ hit the respective transversal cycles in an infinite sequence of points, and we denote the latter by $\left\{x_{n}\right\}_{n=-\infty}^{n=\infty}$ and $\left\{y_{n}\right\}_{n=-\infty}^{n=\infty}$ choosing a proper parametrization (say, by the time). Next we put

$$
\tilde{h}\left(x_{i}\right)=y_{i}, \quad \text { for } \quad i \in \mathbb{Z} .
$$

Because the points $x_{i}$ and $y_{i}$ lie everywhere dense in the respective transversal cycles, we extend by continuity the mapping $\tilde{h}$ to the whole circle, $\tilde{h}: \tilde{C}_{\phi} \rightarrow \tilde{C}_{\psi}$. (Note, that $h$ 'respects' the interval exchange mappings induced on $\tilde{C}_{\phi}$ and $\tilde{C}_{\psi}$ by the flows $\phi^{t}$ and $\psi^{t}$.)

Now we are able to extend the mapping $h$ to the whole fundamental polygon $S_{0}$. It can be done 'in the sense of flow':

$$
\tilde{\phi}^{t}(x)=\tilde{\psi}^{t}(h x), \quad x \in \tilde{C}_{\phi} .
$$

As far as $2 g-2$ saddle points are concerned, it takes more care. (The idea is to follow the respective separatrices.) Now we have an extended mapping $\tilde{h}: S_{0} \rightarrow S_{0}$. Finally, we prolong it to the rest of the Lobachevky plane by the isometries from $\Gamma$. To obtain the required mapping on the surface $M$, it remains to take a quotient $\mathbb{H} / \Gamma$. We claim that the constructed mapping $h$ is a homeomorphism $M \rightarrow M$.

Indeed, in the 'typical' points it follows from the continuity of orbits with regard to the 'initial data'. Some precaution should be taken near the separatrix points. But it is also rather standard; see [1]. The theorem is proved.

Conclusions. A discussion on what one may understand by a linear flow on a compact 2-dimensional manifold of genus $g>1$ is not over; cf. Aranson et al. [1 and Katok-Hasselblatt [7. This question is of top priority because of attempts to push the Kolmogorov-Arnold-Moser (KAM) theory beyond the case $g=1$. We suggest the following definition (see also [10] for connections with the KAM-theory, Closing Lemma and basic sets of $A$-diffeomorphisms):

Definition 2. Let $\alpha$ be a real number and $M$ a compact surface of genus $g>1$. By a linear flow on $M$ one understands flow which is: (i) given by the differential equation $\frac{d x}{d t}=1, \frac{d y}{d t}=\alpha$ w.r.t. every $(x, y)$-chart defined by the lattice $\mathbb{L} \subset \mathbb{H}$; (ii) invariant w.r.t. the isometries $\Gamma$ 's; cf. Definition 1, Clearly, linear flow has the rotation number $\alpha$. 


\section{ACKNOWLEDGMENTS}

I would like to thank a referee for the valuable suggestions and G. Elliott for his steady interest toward this work.

\section{REFERENCES}

[1] S. Kh. Aranson, G. R. Belitsky and E. V. Zhuzhoma, Introduction to the Qualitative Theory of Dynamical Systems on Surfaces, Translations of mathematical monographs, Volume 153, AMS, (1996). MR 97c:58135

[2] K. R. Davidson, $C^{*}$-Algebras by Example, Fields Inst. Monographs 6, AMS 1996. MR 97i:46095

[3] E. G. Effros and C. L. Shen, Approximately finite $C^{*}$-algebras and continued fractions, Indiana Univ. Math. J. 29 (1980), 191-204. MR 81g:46076

[4] G. A. Elliott, On the classification of inductive limits of sequences of semi-simple finite dimensional algebras, J. of Algebra 38 (1976), 29-44. MR 53:1279

[5] T. Giordano, I. F. Putnam and C. F. Skau, Topological orbit equivalence and $C^{*}$-crossed products, J. reine angew. Math. 469 (1995), 51-111. MR 97g:46085

[6] E. Hopf, Ergodentheorie, in: Ergebnisse der Math. und ihrer Grenzgebiete, Bd.5, Springer 1970.

[7] A. Katok and B. Hasselblatt, Introduction to the Modern Theory of Dynamical Systems. (Encyclopedia of mathematics and its applications). Cambridge Univ. Press, 1995.

[8] H. Minkowski, Geometrie der Zahlen, Leipzig, 1910.

[9] P. J. Myrberg, Ein Approximationssatz fur die Fuchsschen Gruppen, Acta Math. 57 (1931), 389-409.

[10] I. Nikolaev, Artin's numbers, CRM-2534, Univ. de Montréal, Preprint (1998); available http://www.crm.umontreal.ca

[11] I. Putnam, The $C^{*}$-algebras associated with minimal homeomorphisms of the Cantor set, Pacific J. of Math. 136 (1989), 329-353. MR 90a:46184

[12] A. Weil, Les familles de courbes sur le tore. Mat. Sbornik 1 (1936), No 5, 779-781.

CRM, Université de Montréal, Montréal H3C 3J7, Canada

E-mail address: nikolaev@crm.umontreal.ca

Fields Institute, 222 College Stree, Toronto, Canada M5T 3J1 